http://jmscr.igmpublication.org/home/ ISSN (e)-2347-176x ISSN (p) 2455-0450 crossref DOI: https://dx.doi.org/10.18535/jmscr/v8i8.08

\title{
Expression of Major Capsid Protein L1 of Human Papilloma Virus Type-16 in Cervical Neoplasia and Its Implications
}

\author{
Authors \\ Ananya Pal ${ }^{1}$, Rajat Kanti Biswas ${ }^{2}$, Pranab Kumar Bhattacharya ${ }^{3}$ \\ ${ }^{1}$ Assistant Professor, Department of Pathology, NRS Medical College, Kolkata, India \\ ${ }^{2}$ Associate Professor, Department of Pharmacology, Malda Medical College, Malda, India \\ ${ }^{3}$ Professor, Department of Pathology, School of Tropical Medicine, Kolkata, India \\ *Corresponding Author \\ Rajat Kanti Biswas \\ Associate Professor, Department of Pharmacology, Malda Medical College, Malda, India
}

\begin{abstract}
Objective: The objective was to investigate the expression of HPV16 L1 capsid protein in cervical neoplasia to analyse its association with different grades of cervical lesions and its implication as a prognostic marker.

Materials and Methods: Detection of L1 capsid protein of HPV16 by immunostaining was done using anti-HPV16 mouse monoclonal antibody against L1 fusion protein in cervical scraping or biopsy specimens obtained from subjects $(n=79)$ after confirmation of cytological/histological diagnosis.

Results: Total 79 cases of cervical cancerous lesions in a graded spectrum were observed for expression of L1 capsid protein of HPV16, among which 30 cases showed L1 expression and 49 cases did not. The expression rates of L1 protein among ASCUS, ASC-H, LSIL and HSIL were $37.50 \%, 66.67 \%, 80 \%$ and $44.44 \%$ respectively. Among invasive lesions, the L1 expression rates were $25 \%, 15.38 \%$ and $8.33 \%$ in well, moderately and poorly differentiated SCC respectively. Overall the L1 positivity rate was $37.97 \%$. The expression of L1 proteins decreased with increase in lesion grade. This declining trend of L1 positivity rate with increasing grade of lesion was extremely significant ( $p$ value for chi squared for trend is 0.0005 ). The difference of L1 expression between preinvasive and invasive group was also significant ( $p<0.0001)$.

Conclusion: Expression of L1 capsid protein of HPV16 was high in lower grade preinvasive lesions and it steadily declined with increasing grade of lesion. HPV16 L1 Protein expression can be used as a prognostic tool and a marker of immune response against HPV16.
\end{abstract}

Keywords: Human Papilloma Virus 16, L1 capsid protein, cervical neoplasia, Immunohistochemistry.

\section{Introduction}

Cancer of the uterine cervix is one of the most important cancers among women. It is the fourth most common cancer in women worldwide ${ }^{[1]}$. In India, 122,844 women are diagnosed with cervical cancer and 67,477 die from the disease every year ${ }^{[2]}$. Cervical cancer is the third largest cause of cancer mortality in India accounting for nearly $10 \%$ of all cancer-related deaths in the country ${ }^{[2]}$.

Human Papilloma Virus (HPV) infection is the major cause for development of cervical carcinoma. The progression of cervical precancerous lesions into cervical cancer is mainly attributed to the sustained infection and integration of high-risk 
$\mathrm{HPV}^{[3]}$. Among the high risk types, HPV type 16 is regarded as the most important in cervical carcinogenesis and is detectable in more than $50 \%$ of cervical cancers ${ }^{[4]}$.

HPV consists of an icosahedral capsid, composed of L1 and L2 capsid/fusion proteins, which enclose a circular double-stranded DNA genome. Major capsid protein L1 constitutes the primary structural element of viral capsid and comprises $90 \%$ of viral surface proteins and is typically expressed during the late phase of viral replication ${ }^{[5]}$. Productive viral infection is characterized by genome amplification and expression of late viral genes responsible for virion assembly, notably the capsid proteins. This late gene expression is restricted to terminallydifferentiated, superficial squamous epithelial cells [6].

The concept of a stepwise development of cervical squamous cell carcinoma was introduced nearly a century ago. The Bethesda system of classification intended for cytology is commonly used now a day. This classifies preinvasive lesions as 'atypical squamous cells of undetermined significance' (ASCUS), 'atypical squamous cells cannot exclude high-grade squamous intraepithelial lesion' (ASC$\mathrm{H})$, 'low-grade squamous intraepithelial lesion' (LSIL) and 'high-grade squamous intraepithelial lesion' (HSIL) as per their progressive $\operatorname{grades}^{[7]}$, which was used in our study. For invasive lesions and histopathological classification we have used common terminology i.e. well differentiated squamous cell carcinoma (WDSCC), moderately differentiated squamous cell carcinoma (MDSCC) and poorly differentiated squamous cell carcinoma (PDSCC) as increasing grades of lesions.

Most of the immunoreactive studies on the HPV 16 L1 capsid protein have demonstrated the relation of expression of the protein with lower grade lesions (ASCUS and LSIL) ${ }^{[8],[9]}$. However, there are very few studies to investigate the role of L1 protein immunoreactivity in higher grade preinvasive (HSIL) and invasive cancerous lesions.

Therefore our study was aimed to find the pattern of expression HPV $16 \mathrm{~L} 1$ capsid protein across the spectrum of cervical squamous cell neoplasia and its possible role as a prognostic marker to predict progression from lower to higher grade lesions.

\section{Materials and Method}

This study was carried out in department of Pathology, Institute of Post Graduate Medical Education and Research (IPGMER), Kolkata, in collaboration with department of Gynaecology \& Obstetrics.

\section{Study Population}

Subjects of our study were recruited from patients attending OPD or admitted in indoor of department of Gynaecology \& Obstetrics, IPGMER, Kolkata.

\section{Sample Size of the Study}

Total 79(seventy nine) subjects were included in this study, which included both preinvasive and invasive lesions of cervix.

\section{Study Duration}

Total period of the study was two years and three months.

\section{Selection of Cases}

\section{Inclusion Criteria}

1. Cytologically suggestive preinvasive and invasive cancerous lesions of cervix.

2. Histologically proven preinvasive and invasive cancerous lesions of cervix.

\section{Exclusion Criteria}

Cytologically and histologically benign lesions of cervix.

\section{Stepwise Method of the Study}

Permission was taken from the institutional ethics committee (IEC) after submitting the synopsis. Informed written consent was also taken from all patients before inclusion. Patients attending OPD or admitted in indoor with chief complains of menstrual abnormalities, excessive/offensive vaginal discharge, pelvic pain, bladder/rectal symptoms etc. were examined and screened. After screening, cervical scraping (for cytology) or punch/surgical biopsy (for histology) were obtained accordingly.

Samples for cytology were divided into two sets. First set was stained with Papanicolaou's (Pap) stain and Leishman-Giemsa stain for cytological examination. The second set was preserved for 
immunocytochemical examinations. Formalin fixed paraffin embedded blocks were prepared from biopsy specimens and 3 to 4 micron thick serial sections were made. Here also, two sets of slides were prepared. First set was stained with Hematoxylin \& Eosin stain for histological examination. The second set was preserved for immunohistochemical (IHC) staining. After microscopical examination, the smears of cytologically suggestive cancerous lesions and the sections of histologically proven preinvasive and invasive malignant lesions were recruited in the study for immunocytochemical/ immunohistochemical staining.

\section{Immunostaining}

Principle: The demonstration of a specific substance by immunostaining is a two-step process involving first, the binding of a primary antibody to the substance (antigen) of interest and second, the detection of bound antibody by a chromogen. Antibodies are raised artificially against a specific antigen. Those antibodies bind to that specific antigen, if it is present in the tissue section or smear on study. The antigen-antibody complex is then visualized using a immunochemical method, e.g. antibodies against initial antibody and a dye complex i.e. diaminobenzidine in immunoenzymatic method. The immunoenzymatic method is commonly based on four techniques i.e. peroxidase, biotin-avidin, alkaline phosphatase and gold-silver technique. In this study we have used 'BioGenex' anti-HPV-16 mouse monoclonal antibody from ascitic fluid diluted in phosphate buffered saline, $\mathrm{pH}$ 7.6, containing $1 \%$ BSA and $0.09 \%$ sodium azide $^{[10]}$. This antibody, of immunoglobulin class IgG2a, has been developed against immunogen 'Beta-galactosidase-L1 fusion/L1 capsid proteins' of HPV-16 (catalog no. AM362-5M) and the clone used is Cam Vir-1 (catalog no. MU362-UC) with protein concentration 10-15 $\mathrm{mg} / \mathrm{ml}$. For demonstration of the antigen-antibody complex, we have used 'The Supersensitive Polymer-HRP Detection System'. It is a novel detection system based on a non-biotin polymeric technology that uses two major components: super enhancer and a
poly-HRP reagent. The secondary antibody used here is conjugated with Horseradish Peroxidase polymer (HRP) and 3 3' Diaminobenzidine Tetrahydrochloride (DAB) substrate or ACE substrate.

Method: Sections of tissue or smears were drawn over Poly-L-Lysine coated slides. Deparaffinization of paraffin embedded sections was done with xylene followed by rehydration with descending grade of alcohol. Antigen retrieval was done with citrate buffer ( $\mathrm{pH} 2.5$ ) in microwave and blocking reagent $\left(\begin{array}{lll}3 \% & \mathrm{H}_{2} \mathrm{O}_{2}\end{array}\right)$ was added with interval washing. Primary antibody (Anti HPV16 Mouse Monoclonal Antibody) was added followed by addition of secondary antibody (Super Enhancer ${ }^{\mathrm{TM}}$ reagent) with required buffer washing. Subsequently HRP and DAB were added. Counter staining with Harris's Haematoxylin, dehydration and finally mounting was done. One positive and one negative control were used for assessment of proper immunostaining.

Interpretation: Positive staining with mouse monoclonal antibody against HPV16 L1 fusion/capsid protein was demonstrated by brown coloured nuclear and/or cytoplasmic stain.

\section{Statistical Analysis}

Chi-squared test for trend was used to determine if there was a significant trend in the proportion of cases expressing L1 capsid protein across the spectrum of the ordered categories of lesions according to their grade. Fisher's exact test and Odd's ratio was calculated to compare the difference of L1 expression between preinvasive and invasive group. The level of significance was $\mathrm{p}<0.05$. The statistical analysis was conducted using GraphPad Prism Version 7.0.

\section{Result}

The expression of $\beta$-galactosidase HPV16 L1 fusion/capsid protein was observed by a strong nuclear and sometimes cytoplasmic staining of cytological and histological specimens of preinvasive and invasive cervical cancerous lesions following immunostaining with mouse monoclonal antibodies. 
LSIL (fig. 4) showed L1 expression by nuclear positivity in the basal layers, whereas HSIL (fig. 3) showed strong nuclear and cytoplasmic positivity mainly in the basal and middle layers but sometimes in all the three layers. Strong cytoplasmic expression and weak nuclear staining in malignant cells was suggestive of presence of HPV16 L1 protein in invasive (higher grade) SCC by IHC (fig. 5).

We tabulated different cervical cancerous lesions (preinvasive and invasive) in a graded manner (from lower to higher grade) to observe L1 expression positivity rate.

Total 79 cases of cervical cancerous lesions were observed for expression of major capsid protein L1 for HPV16, within which the number of ASCUS, ASC-H, LSIL, HSIL, WDSCC, MDSCC and PDSCC cases were 8, 6, 15, 9, 16, 13 and 12 respectively.

Among the total 79 cases 30 cases showed positive L1 expression and 49 cases did not expressed L1. The expression rates of HPV16 L1 protein in preinvasive lesions among ASCUS, ASC-H, LSIL and HSIL were $37.50 \%, 66.67 \%, 80 \%$ and $44.44 \%$ respectively. Among invasive lesions the L1 expression rates were $25 \%, 15.38 \%$ and $8.33 \%$ in WDSCC, MDSCC and PDSCC respectively. Overall L1 positivity rate was $37.97 \%$ in all the lesions (table-1, fig. 1).

The expression of L1 capsid proteins decreased with increase in lesion grade (though ASCUS and ASC$\mathrm{H}$ showed a bit lower L1 positivity). Maximum L1 expression was found among LSIL lesions (80\%) whereas PDSCC showed minimal L1 expression (8.33\%) (fig. 2). The $\mathrm{p}$ value for chi squared for trend is 0.0005 (table-1). This signifies that the decreasing trend of L1 positivity in graded spectrum (from lower to higher grade) of cervical cancerous lesion is extremely significant.

When we compared the expression of L1 protein between preinvasive $(60.53 \%$ positivity) and invasive (17.07\% positivity) group, the difference of expression was extremely significant ( $\mathrm{p}$ value $<0.0001$, OR-7.448, $95 \% \mathrm{CI}=2.628$ to 21.110 ) (table-2).
Table 1: Demonstration of HPV16 L1 protein expression among different grades of cervical cancerous lesions:

\begin{tabular}{|l|c|c|c|c|}
\hline LESION & $\begin{array}{c}\text { No. of } \\
\text { Cases }\end{array}$ & $\begin{array}{c}\text { L1 } \\
\text { Positive }\end{array}$ & $\begin{array}{c}\text { L1 } \\
\text { Negative }\end{array}$ & $\begin{array}{c}\% \\
\text { Positive }\end{array}$ \\
\hline ASCUS & 8 & 3 & 5 & $37.50 \%$ \\
\hline ASC-H & 6 & 4 & 2 & $66.67 \%$ \\
\hline LSIL & 15 & 12 & 3 & $80 \%$ \\
\hline HSIL & 9 & 4 & 5 & $44.44 \%$ \\
\hline WDSCC & 16 & 4 & 12 & $25 \%$ \\
\hline MDSCC & 13 & 2 & 11 & $15.38 \%$ \\
\hline PDSCC & 12 & 1 & 11 & $8.33 \%$ \\
\hline TOTAL & 79 & 30 & 49 & $37.97 \%$ \\
\hline
\end{tabular}

$*$ Chi Squared for trend $=12.201$

$* \mathrm{p}$ value $=0.0005$

* Significant linear trend of L1 Positivity among different groups of cervical cancerous lesions in graded spectrum

Table 2: Difference of HPV16 L1 protein expression between pre-invasive $\&$ invasive lesions of cervix:

\begin{tabular}{|l|c|c|c|c|}
\hline LESION & $\begin{array}{c}\text { No. of } \\
\text { Cases }\end{array}$ & $\begin{array}{c}\text { L1 } \\
\text { Positive }\end{array}$ & $\begin{array}{c}\text { L1 } \\
\text { Negative }\end{array}$ & $\begin{array}{c}\% \\
\text { Positive }\end{array}$ \\
\hline Preinvasive & 38 & 23 & 15 & $60.53 \%$ \\
\hline Invasive & 41 & 7 & 34 & $17.07 \%$ \\
\hline Total & 79 & 30 & 49 & $37.97 \%$ \\
\hline
\end{tabular}

$*$ Odds ratio $(\mathrm{OR})=7.448$

$* 95 \% \mathrm{CI}=2.628$ to 21.110

$*$ p value $<0.0001$

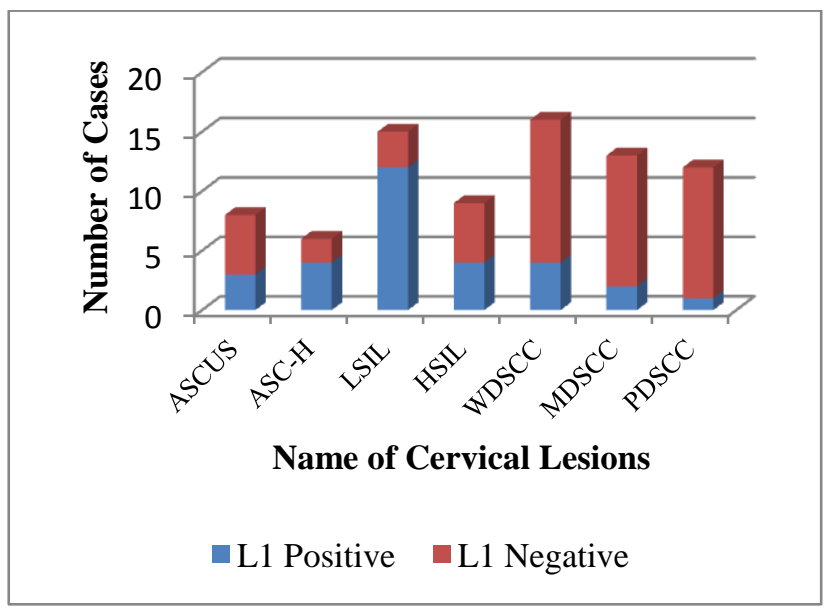

Fig. 1 Bar diagram showing L1 positive and negative case distribution among different grades of cervical lesions 


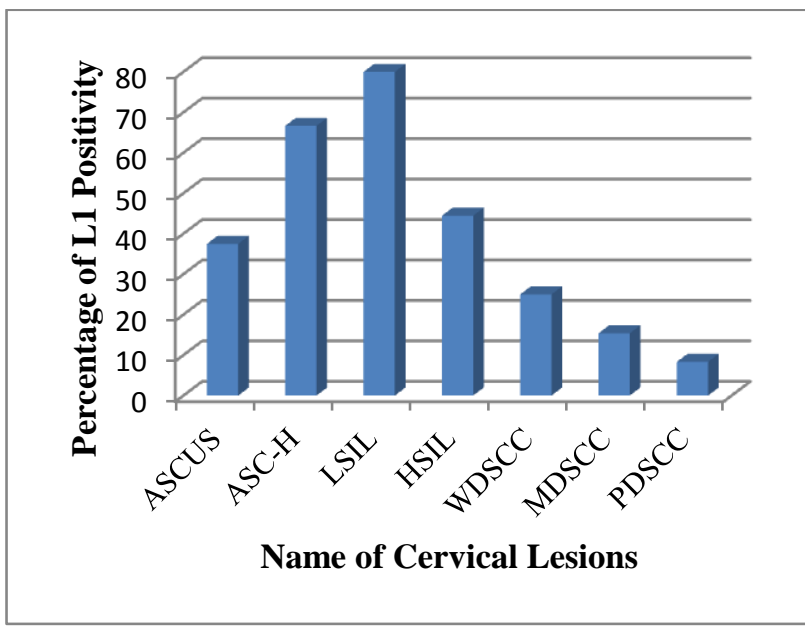

Fig. 2 Bar diagram showing L1 expression positivity rate among different cervical lesions

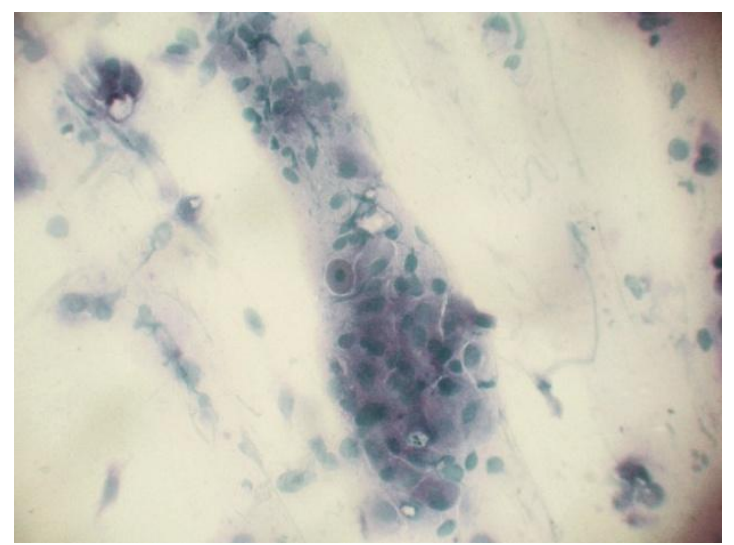

Fig. 3 Positive cytoplasmic staining for HPV L1 capsid protein in HSIL (Cytology)

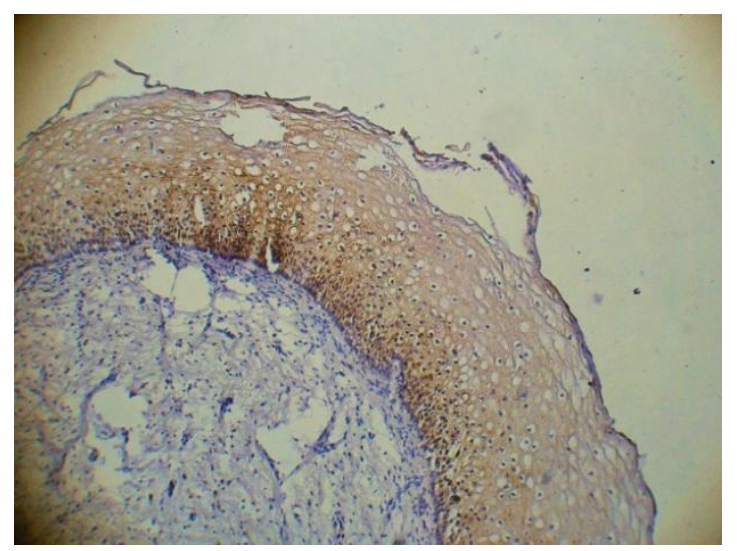

Fig. 4 Positive nuclear and cytoplasmic staining for HPV L1 capsid protein in basal epithelium of LSIL (Histology)

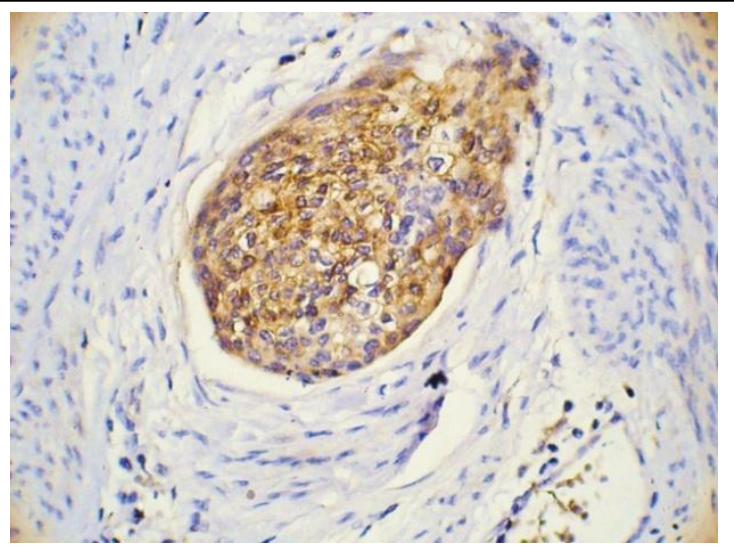

Fig. 5 Positive nuclear and cytoplasmic staining for HPV L1 capsid protein in invasive SCC (Histology)

\section{Discussion}

Our study showed that expression of L1 capsid protein of HPV16 by immunocytochemistry / immunohistochemistry has a typical trend. The L1 expression is higher in lower grade lesions and lower in higher grade lesions, maintaining a linear trend where L1 capsid protein expression tends to decline with increasing severity of the lesions. In our study though ASCUS and ASC-H showed lower L1 expression than LSIL, which can be explained by a wide variety of histopathology of ASCUS and ASC-H ranging from reactive changes to carcinoma. Overall the preinvasive lesions showed significant increased expression of L1 than invasive lesions. Our study findings corroborates with the previous studies, where declining L1 positivity with higher grade of lesions found, though the positivity rates differs in different studies ${ }^{[6],[11],[12],[13]}$.

In cervical tissue of low-grade lesions, HPV usually exists in an episomal state in the host nucleus and proceeds into the productive cycle. The productive cycle is composed of HPV infection of the basement membrane and transcription, translation, and assembly of the L1 capsomere in the epithelium ${ }^{[14]}$. Ultimately, it produces L1 capsid protein in the superficial layers of the cervical epithelium ${ }^{[14]}$. On the other hand, in cases with high-grade preinvasive or invasive SCC, the E2 open reading frame of HPV DNA is frequently disrupted and integrated into the human genome. The loss of E2 inhibitory function produces E6 and E7 oncoproteins and causes suppression of tumor suppressor proteins p53 and $\mathrm{Rb}^{[14]}$. In abortive HPV cycles of high-grade lesions, 
HPV L1 cannot be detected at the superficial epithelium $^{[13]}$.

Loss of L1 protein expression in HPV16 infected cervical tissue may be due to two reasons. First, there is integration of viral DNA into the host genome and secondly, the latent/persistant infection with low or no synthesis of HPV oncoprotein and no HPV production in the long-living basal epithelial cells $^{[13]}$. The decreased expression of L1 protein in higher grade lesions, found in our study, can be explained by this.

The HPV16 L1 protein is immunogenic to CD8+ and CD4+ $\mathrm{T}$ cells, which play important roles in cellular immunity ${ }^{[15]}$. HPV L1-negative lower grade lesions are reportedly more likely to progress to higher grades than L1-positive cases; this suggests that HPV L1 has a beneficial immunological role in the prevention of disease progression ${ }^{[16]}$. Therefore, it is presumed that, the absence of HPV L1 after HPV integration into the host genome is associated with immunologic evasion in higher grade lesions [15],[17]. The presence of HPV L1 could be an indicator of a defence status in HPV infection. This immunological background of L1 expression has definitely given it a prognostic important.

Another important point about L1 expression is that, lack of L1 capsid protein may reflect abnormalities in transcription-factor pathways that could be responsible for the disturbed basal epithelial cell maturation and ultimately disease progression ${ }^{[16]}$. The presence of HPV L1 presumably represents the episomal stage of a transient HPV infection and correlates well with the regression of the abnormal pathology, whereas loss of HPV L1 is associated with a higher risk of progression to invasive lesions [12].

In 2003, Melsheimer $\mathrm{P}$ et $a l .{ }^{[17]}$ demonstrated that expression of L1 capsid protein is significantly reduced in HPV16 infected higher grade lesions, though lower grade lesions are expressing it, which finding is consistent with our present study. They have also shown that absence of L1 expression may be used as a predictor of persistence or progression to higher grade lesions and L1 capsid protein could function as prognostic marker.
Till date there are very few studies to show the prognostic significance of L1 capsid protein in cervical neoplasm ${ }^{[17],[18]}$.

From the above discussion we can come to the following inferences about implications of expression of L1 capsid protein in cervical neoplasia:

We can routinely perform HPV16 L1 protein expression along with cervical cytology in earlier/preinvasive lesions. HPV L1 positivity can suggest a good defence against HPV infection, on the other hand lack of L1 capsid protein may be a marker of reduced cellular immune responses, thereby promoting further transformation of immature epithelial cells. The immunostaining of L1 capsid protein could be useful particularly in the lower grade / early preinvasive lesions, because its expression status might be able to identify individuals at risk of lesion progression and be helpful to determine the need of subsequent followup. Therefore, we can consider the L1 capsid protein expression by immunostaining as a very useful and powerful prognostic marker.

The limitations of our study were, firstly it was not a prospective study, hence follow up was not possible and secondly, expression of p16 was not done, which could be an important prognostic marker when done along with L1.

\section{Conclusion}

To summarise, our study demonstrated that, expression of L1 capsid protein of HPV16 was high in lower grade preinvasive lesions and it steadily declined with increasing grade of lesion. This trend was statistically significant. The progression of preinvasive to invasive lesions is associated with corresponding reductions in L1-capsid protein expression. Therefore, HPV16 L1 Protein expression can be used as a very important prognostic tool and a marker of immune response, the absence of which may suggest enhanced malignant potential. 


\section{Acknowledgement}

We are acknowledging the support and helps of the Department of Pathology and Department of Gynaecology \& Obstetrics, IPGMER, Kolkata.

\section{References}

1. Ferlay J, Soerjomataram I, Ervik M, Dikshit $\mathrm{R}$, Eser S, Mathers C, et al. GLOBOCAN 2012 v1.0, Cancer Incidence and Mortality Worldwide: IARC CancerBase No. 11. Lyon, France: International Agency for Research on Cancer; 2013. Available from: http://www.globocan.iarc.fr. [Last accessed on 2013 Dec 16].

2. ICO Information Centre on HPV and cancer (Summary Report 2014-08-22). Human Papillomavirus and Related Diseases in India. 2014.

3. Longworth MS, Laimins LA. Pathogenesis of human papillomaviruses in differentiating epithelia. Microbiol Mol Biol Rev. 2004;68:362-72.

4. Bosch FX, Manos MM, Munoz N, et al. Prevalence of human papillomavirus in cervical cancer: a worldwide perspective. International biological study on cervical cancer (IBSCC) Study Group. J Natl Cancer Inst. 1995;87:796-802.

5. Gu W, Ding J, Wang X, et al. Generalized substitution of isoencoding codons shortens the duration of papillomavirus L1 protein expression in transiently gene-transfected keratinocytes due to cell differentiation. Nucleic Acids Res. 2007;35:4820-32.

6. Yemelyanova A, Gravitt PE, Ronnett BM, Rositch AF, Ogurtsova A, Seidman JD, Roden RBS. Immunohistochemical detection of Human Papillomavirus capsid proteins L1 and L2 in squamous intraepithelial lesions: Potential utility in diagnosis and management. Mod Pathol. 2013 February ; 26(2): 268-274.

7. Solomon D,Davey D,Kumaran $\mathrm{R}$ et al. The 2001 Bethesda System: terminology for reporting results of cervical cytology. JAMA, 2002; 287: 2114-19.

8. Lee SJ, Lee AW, Kim TJ, et al. Correlation between immunocytochemistry of human papilloma virus L1 capsid protein and behaviour of low-grade cervical cytology in Korean women. J Obstet Gynaecol Res. 2011;37:1222-8.

9. Griesser H, Sander H, Walczak C, Hilfrich RA. HPV vaccine protein L1 predicts disease outcome of high-risk HPV+ early squamous dysplastic lesions. Am J Clin Pathol. 2009;132:840-5.

10. Anti-Papillomavirus type 16 (HPV-16) [Cam Vir-1]; Doc. No. 932-362M-4 Rev. D;May 20, 2004.

11. Lee SJ, Lee AW, Kang CS. et al. Clinicopathological implications of human papilloma virus (HPV) L1 capsid protein immunoreactivity in HPV16-positive cervical cytology. Int J Med Sci. 2014;11(1):80-86.

12. Ungureanu C, Socolov D, Anton G, Mihailovici MS, Teleman S. Immunocytochemical expression of p16INK4a and HPV L1 capsid proteins as predictive markers of the cervical lesions progression risk. Rom J Morphol Embryol. 2010;51:497-503.

13. Yoshida $\mathrm{T}$, Sano $\mathrm{T}$, Kanuma $\mathrm{T}$, et al. Immunochemical analysis of HPV L1 capsid protein and p16 protein in liquid-based cytology samples from uterine cervical lesions. Cancer. 2008;114:83-8.

14. Middleton K, Peh W, Southern S, et al. Organization of human papillomavirus productive cycle during neoplastic progression provides a basis for selection of diagnostic markers. J Virol. 2003;77:10186201.

15. Steele JC, Roberts S, Rookes SM, Gallimore PH. Detection of CD4(+)- and CD8(+)-Tcell responses to human papillomavirus type 1 antigens expressed at various stages of the virus life cycle by using an enzyme-linked 
immunospot assay of gamma interferon release. J Virol. 2002;76:6027-36.

16. Griesser H, Sander H, Hilfrich R, Moser B, Schenck U. Correlation of immunochemical detection of HPV L1 capsid protein in pap smears with regression of high-risk HPV positive mild/moderate dysplasia. Anal Quant Cytol Histol. 2004;26:241-245.

17. Melsheimer P, Kaul S, Dobeck S, Bastert G. Immunocytochemical detection of HPV high-risk type L1 capsid proteins in LSIL and HSIL as compared with detection of HPV L1 DNA. Acta Cytol.2003;47:124-8.

18. Griesser H, Sander H, Walczak C, Hilfrich $\mathrm{R}$, Immunochemical detection of HPV-L1 capsid: a prognostic marker for early squamous lesions of the cervix, Acta Cytologica, 2007, 51(2 Suppl):268. 